\title{
Cooperative Transmission Schemes for Energy Efficient Collaborative Wireless Sensor Networks
}

\author{
Muhammad K. Naeem, Mohammad Patwary, Abdel-Hamid Soliman \\ and Mohamed Abdel-Maguid ${ }^{\ddagger}$ \\ m.k.naeem@staffs.ac.uk,m.n.patwary@staffs.ac.uk,a.soliman@staffs.ac.uk and \\ m.maguid@ucs.ac.uk
}

Faculty of Computing, Engineering, and Sciences (FCES), Staffordshire University, Stafford, ST18 0AD, United Kingdom.

${ }^{\ddagger}$ School of Science, Technology and Health

Unversity Campus Suffolk (UCS), Ipswich, IP4 1QJ, United Kingdom.

\begin{abstract}
Energy conservation is one of the prime concerns that leads the researcher to investigate collaborative wireless sensor networks with some application specific challenges. Such challenges include combining distributed data synchronously, performing power aware signal processing, defining communication methods that can provide progressive accuracy and, optimizing processing and communication for signal transmission. A cooperative resource selection and transmission scheme is proposed to improve the performance of collaborative wireless sensor networks in terms of maintaining link reliability. A measure of Channel Quality Index (CQI) is also proposed to obtain dynamic adaptivity and to optimize resource usage within wireless sensor networks according to environment conditions. Based on CQI, a subset of nodes is proposed to be chosen to perform cooperative transmission by exploiting collaboration between wireless sensing nodes. As part of the proposed cooperative nature of transmission, the recently proposed transmit-receive antenna selection scheme and lattice reduction algorithm have also been considered. It is assumed that channel state information (CSI) is estimated at receiver and also there is a feedback link between the wireless sensing nodes and the fusion center receiver. From the simulation results it is observed that for $99.99 \%$ detection reliability, the proposed adaptive transmission scheme and proposed hybrid scheme consume only $15 \%$ and $18 \%$ of energy respectively as compared to the conventional cooperative transmission.
\end{abstract}

Keywords: Wireless Sensor Networks, Virtual MIMO, Collaborative Sensor Networks, Cooperative Transmission, Green Wireless Communication, Adaptive Resource Selection, Lattice Reduction, Channel Quality Index. 


\section{INTRODUCTION}

Wireless sensor networks commonly deployed to serve various applications in the field of security, environmental monitoring, health monitoring, energy as well as battlefield monitoring etc. Wireless sensor networks are usually composed of a number of wireless sensing nodes which collectively monitor and distribute information to the desired destinations. A wireless sensor node is a battery powered device which is expected to perform the tasks that includes sensing of physical quantities, signal processing, computation, data storage and wireless communication. Wireless sensor nodes may be deployed in a hostile environment which make it difficult to change or recharge the batteries of sensor nodes. Recently researchers are exploring a new area of research called green wireless communication. The purpose of green communication is energy conservation and improved renewable energy source utilization as discussed in [1,2]. Energy harvesting from such renewable sources have limited energy generation capacity; and defines the lifetime of a sensing node if dependent of such energy sources. Subsequently, having enhanced lifetime of sensor nodes is one of the key design constrains, while designing wireless sensor networks regardless of the application; without compromising the required quality of service e.g. detection reliability [3].

Ideally wireless sensing nodes are expected to perform the required tasks collaboratively to achieve a common objective. The main idea of such collaboration is either to involve an optimized number of sensing nodes while reporting an incident or to select a set of transmission scheme which involves minimum consumption of energy without compromising quality of service. Recently, significant amount of research has been carried out on sensing node selection while exploiting the advantages provided by the multiple sensor nodes involved in transmission and reception. While optimizing wireless sensor networks, the key challenge is the selection of principal sensing node as well as sink node that provide with assurance of optimum utilization of radio resources as discussed in $[4,5]$. Network specific optimization 
has been proposed with collaborative information sharing scheme as proposed in [6-8] by sub-dividing the whole network into multiple clusters, where each cluster consists of relatively small number of sensors compared to the total number of sensors within the network. For each cluster, a node is proposed to be selected as cluster head that process the required data for the respective cluster. Several other cluster based collaboration schemes are proposed in [9-11] that tries to provide real time processing while using minimum physical resources as well as requiring minimal processing. However, such high dependency on a single node may lead link reliability at risk in severe network conditions such as least amount of available energy at node or deep channel fading etc. Subsequently, having more than a single representative from each cluster come to the attention of the researchers to provide transmit-receive diversity as Multiple-Input Multiple Output (MIMO) based communication schemes.

Significant amount of research has been conducted in [12,13] for MIMO based communication architectures to improve the signal detection reliability, spectral efficiency and information capacity without increasing the transmit power or bandwidth as compared to single antenna systems. Traditionally multiple antennas have been used to achieve transmit or receive diversity to combat fading or to achieve spatial multiplexing to increase the data rate by transmitting the independent information streams through the spatial channels as discussed in $[14,15]$. Beam-forming technique within wireless sensor networks has been proposed in [16] for directional signal transmission and reception that decreases co-channel interference in MIMO systems. Channel adaptive processing intelligence schemes such as lattice reduction, sphere decoding etc. have been proposed in the literature [17-19] for MIMO systems to help the receiver to perform near optimal data detection. Optimal maximum likelihood detection with sphere decoding can achieve full diversity but less complex suboptimal detectors with lattice reduction perform close to optimal and have the potential to achieve full diversity [20].

In the context of wireless sensor networks, several sensor nodes are expected to achieve a 
virtual MIMO system, that can obtain all the benefits of both transmit-receive diversity as well as spatial multiplexing as attainable with conventional MIMO based communication systems as discussed in [21,22]. Several collaborative beam-forming techniques have been proposed in [23-25] that provide solution for coordination and phase synchronization of the sensor nodes such that the signal can be received constructively at the sink nodes with minimal co-channel interference. Cooperative transmission scheme along with collaborative beam-forming is proposed in [26] to create a virtual MIMO system, which has claimed to have higher spectral efficiency and detection reliability due to its spatial multiplexing capability and existence of reduced co-channel interference respectively.

The available power within a sensing node is inversely proportional to the uncertainty of the channel propagation condition, with reference to the budgeted consumption of power as designed. Wireless sensor networks are expected to be adaptive with the dynamic Radio Frequency (RF) propagation environment conditions, while demanded or allocated with resources (e.g. physical resources, processing intelligence etc.) to ensure quality of service based on application requirements. The required quality of service defined in terms of error rate, delay and degree of information security. The quality of service can be guaranteed by exploiting effective link adaptation scheme. The purpose of link adaptation is to select appropriate physical resources and processing intelligence schemes that are best suited to the channel conditions to offer quality of service based on application requirements. Channel selection schemes for efficient and reliable data transmission within wireless sensor networks have been proposed in $[27,28]$, which are based on channel's link quality and it is assumed that each node has multiple antennas.

Without having any exception from any wireless communication, wireless sensor networks are also expected to provide maximum transmit-receive reliability with optimum usage of radio resources e.g. power, bandwidth etc. To obtain maximum optimization performance, 
explicit or implicit knowledge of the channel quality features at transmitter is required. Hence, classification of such channel quality features as estimated at the receiver can be fed back to the transmitter with negligible spectral resources is required. Within the scope of author's knowledge, limited research can be found in the literature. In this paper, Cooperative resource selection and transmission scheme has been considered to achieve virtual MIMO communication for wireless sensing nodes. A framework has been proposed to utilize minimum resources and processing intelligence to guarantee the required quality of service. It is assumed that the channel state information is perfectly estimated at the receiver. An adaptive channel quality indexing scheme is proposed in the context of wireless sensor networks. On the basis of such classification a dynamic allocation of radio and physical resources as well as selection of deployable processing intelligence has been proposed. The rest of the paper is presented in the following order. A system model is described in section II; cooperative transmission and adaptive optimization schemes for wireless sensor networks are presented in section III; Simulation results have been presented in section IV along with the comparison of existing frameworks to evaluate the performance of the proposed framework followed by conclusion in section $\mathrm{V}$.

\section{SYSTEM MODEL}

The system model considered in this paper is shown in Fig. 1. A wireless sensor network with $n$ number of non-uniformly distributed sensor nodes is assumed. Energy conservation is a key issue within wireless sensor network and it is expected to be achieved by optimizing the network e.g. by introducing cooperative transmission schemes within wireless sensor networks. Cooperation is expected to be defined by observing channel quality based on fading or interference depth while transmitting to fusion center. To attain collaborative optimization, collaboration criterion is to be defined by mutual agreement between candidate transmitting sensor nodes and fusion center. To achieve such optimization in the proposed scheme, channel state information is required at transmitting sensor nodes. It is assumed that all the sensor 
nodes in the network are capable of wireless transmission and reception, and channel is state information is known to candidate transmitting sensor nodes through a feedback link from fusion center. It is expected that the collaboration is to be performed by the network. Let $A$ is a set a of all the sensor nodes in the network which is defined as:

$$
A \in\left\{S_{1}, S_{2}, \ldots, S_{n}\right\}
$$

Where $S(\cdot)$ represents the sensor nodes. The network is divided into $q$ number of clusters, where each cluster consists of $n_{p}$ number of sensor nodes, then $n_{p}=n / q$. Let $Q$ represents the set of sensor nodes within a cluster, which is defined as:

$$
Q \in\left\{S_{1}, S_{2}, \ldots, S_{n_{p}}\right\}
$$

Each cluster has its own predefined cluster head which is responsible for collaboration between the sensor nodes within the cluster to achieve reliability. Although the cluster heads are predefined, the number of sensors reporting to the cluster heads, which are belong to a cluster, is defined by the occurrence location of an incident. Subsequently, the number of sensor nodes within a cluster, as denoted earlier with $n_{p}$ becomes a random variable within our proposed algorithm. Due to the co-operative nature of the proposed algorithm, the sensor nodes included within a cluster for reporting an incident may vary from one incident to another; on the basis of the distance of the sensor nodes from the reportable incident location, respective cluster head location as well as the acquired signal strength induced by the incident. Apart from this, due to the reconfigurable nature of the proposed algorithm, the range of the number of sensor nodes to be included within a cluster is bounded by a set of maxima and minima as denoted by $\left(n_{p}^{\max }, n_{p}^{\min }\right)$ respectively; i.e. $n_{p} \in\left\{n_{p}^{\min }: n_{p}^{\max }\right\}$. Cluster heads are also responsible for communication with the rest of the other cluster heads and fusion center receiver to achieve spatial multiplexing. It is assumed that the communication protocol to be assumed within the cluster is star and communication protocol between the cluster heads is mesh. Let $N_{t}$ denotes the number of cluster heads involved in transmission, where $N_{t} \leq q$. The transmitted data 
vector $\mathbf{x}$ is defined as:

$$
\mathbf{x}=\left[x_{1}, x_{2}, \ldots, x_{N_{t}}\right]^{T}
$$

The received signal vector at fusion center can be expressed as:

$$
\mathbf{y}=\mathbf{H x}+\mathbf{n}
$$

Assuming $N_{r}$ number of receiving antennas at the fusion center receiver, $\mathbf{y}$ is the received signal vector with dimensions $\left(N_{r} \times 1\right), \mathbf{H}$ is the Rayleigh fading channel matrix of size $\left(N_{r} \times N_{t}\right)$ and $\mathbf{n}$ is the noise vector with dimensions $\left(N_{r} \times 1\right)$. The noise is considered to be additive white Gaussian noise with zero mean and unity variance $\sigma^{2}$. The Rayleigh fading channel matrix is defined as:

$$
\mathbf{H}=\left[\begin{array}{ccc}
h_{1,1} & \ldots & h_{1, N_{t}} \\
\vdots & \ddots & \vdots \\
h_{N_{r}, 1} & \ldots & h_{N_{r}, N_{t}}
\end{array}\right]
$$

Where $h_{j, i}$ denotes the channel coefficients from $i^{\text {th }}$ transmitter sensor node to $j^{\text {th }}$ receiving antenna at the fusion center with $i \in\left\{1,2, \ldots, N_{t}\right\}$ and $j \in\left\{1,2, \ldots, N_{r}\right\}$ respectively. Both the sensor node and the fusion center receiver are assumed to be stationary. Hence slow fading wireless channel is expected to be observed by the receiver. Lets assume, $\sigma_{h}^{2}$ denotes the variance of the Gaussian channel coefficients within channel matrix $\mathbf{H}$ when $i=j$ and $\sigma_{s}^{2}$ denotes the variance of the Gaussian channel coefficients within channel matrix $\mathbf{H}$ when $i \neq j$. Hence, the degree of sharpness of beam-forming is represented by signal to leakage ratio (SLR), where

$$
S L R=\frac{\sigma_{h}^{2}}{\sigma_{s}^{2}}
$$

That is, the higher the value of SLR, the sharper is the beam with respect to the correlated component. It is assumed that $\left(\sigma_{h}^{2}>\sigma_{s}^{2}\right)$. 


\section{PROPOSED OPTIMIZATION AND ADAPTATION SCHEME}

To conserve energy of sensor nodes within wireless sensor network, it is expected to select optimum number of sensor nodes to transmit the data to fusion center. In this paper, a cooperative transmission scheme is proposed, which is expected to optimize the network. Energy conservation is expected to be achieved by selecting optimum number of sensor nodes for transmission with reference to some predefined criterion. Transmit diversity or spatial multiplexing are expected to be achieved because of the cooperation between the sensing nodes.

\section{A. Optimization Scheme}

An optimized criterion to select resources which are expected to cooperate while transmission is defined as:

$$
\zeta=\min \left[\frac{1}{n_{r} n_{t}} \sum_{u=1}^{n_{r}} \sum_{v=1}^{n_{t}}\left(\mathbf{H}_{S(u, v)} \mathbf{H}_{S(u, v)}^{H}-\mathbf{I}_{(u, v)}\right)^{2}\right]
$$

Where $\mathbf{H}_{S}$ is the channel matrices of the possible combinations of transmit-receive antennas and $\mathbf{I}$ is the identity matrix. Network optimization is also expected to be achieved by defining collaboration between clusters. It is assumed that the fusion center receiver cooperates with the clusters through a feedback link as shown in Fig. 1. It is intended to design a transmit filter and the design criterion to be selected based on feedback information from fusion center receiver. The transmit filter is designed with the aim of minimizing the affect of leakage interference on the signal. Lenstra-Lenstra-Lovasz (LLL) lattice basis reduction

algorithm is considered to determine a corresponding reduced basis $\widetilde{\mathbf{H}}$ with better properties by searching for the reduced lattice basis of the lattice defined by the channel matrix $\mathbf{H}$. The Fig. 2 shows the block diagram for a MIMO system with lattice reduction added detection. 
The received signal $\mathbf{Y}$ at fusion center receiver is defined as:

$$
\mathbf{Y}=\mathbf{H T X}+\mathbf{N}
$$

Where

$$
\mathbf{T}=\mathbf{H}^{H} \widetilde{\mathbf{H}}
$$

Where $\widetilde{\mathbf{H}}$ is obtained from LenstraLenstraLovsz (LLL) lattice basis reduction algorithm. Let $\mathbf{H}=\left[\mathbf{h}_{1} \mathbf{h}_{2} \ldots \mathbf{h}_{N_{t}}\right]$, where $\mathbf{h}_{i}$ are the column vectors of $\mathbf{H}$. The LLL Lattice reduction algorithm is summarized in the Table. I.

TABLE I

LLL LAtTice Reduction Algorithm

\section{1: $\widetilde{\mathbf{H}} \leftarrow \mathbf{H}$}

2: $[\widetilde{\mathbf{Q}}, \widetilde{\mathbf{R}}] \leftarrow q r(\widetilde{\mathbf{H}})$

$\mathbf{3}: \mathbf{T} \leftarrow \mathbf{I}_{m}$, where $\mathrm{m}$ is number of columns of $\mathbf{H}$

4: $l \leftarrow 2$

5: $\frac{1}{4}<\delta<1$

6 : repeat

$\mathbf{7}: \mu=\left\lceil\frac{\widetilde{\mathbf{R}}(l-1, l)}{\widetilde{\mathbf{R}}(l-1, l-1)}\right\rfloor$

$8: \widetilde{\mathbf{H}}_{l} \leftarrow \widetilde{\mathbf{H}}_{l}-\mu \widetilde{\mathbf{H}}_{l-1}$

$\mathbf{9}: \zeta=\|\widetilde{\mathbf{R}}(l, l)+\widetilde{\mathbf{R}}(l-1, l)\|^{2}$

$\mathbf{1 0}:$ if $\delta|\widetilde{\mathbf{R}}(l-1, l-1)|^{2}>\zeta$

11 : Swap columns $l-1$ and $l$ in $\widetilde{\mathbf{H}}, \widetilde{\mathbf{R}}$ and $\mathbf{T}$

12 : Calculate rotation matrix $\Theta$ such that the elements $\widetilde{\mathbf{R}}(l, l-1)=0$

$$
\Theta=\left[\begin{array}{cc}
\alpha & \beta \\
-\beta & \alpha
\end{array}\right] \quad \text { with } \quad \alpha=\frac{\widetilde{\mathbf{R}}(l-1, l-1)}{\zeta}, \quad \beta=\frac{\widetilde{\mathbf{R}}(l, l-1)}{\zeta}
$$

$13: \widetilde{\mathbf{R}}(l-1: l, l-1: m)=\mathbf{\Theta} \widetilde{\mathbf{R}}(l-1: l, l-1: m)$

$\mathbf{1 4}: l \leftarrow \max (l-1,2)$

15 : else

16: $l \leftarrow l+1$

17 : end if

18 : untill $l>m$

Lattice reduction is expected to achieve high detection reliability as compared to proposed resource selection scheme but it requires high computational complexity. On the other side, 
proposed resource selection scheme is expected to be energy efficient. To achieve high detection reliability while minimizing energy consumption, a hybrid scheme is proposed. The hybrid scheme is combination of resource selection and lattice reduction schemes. The hybrid scheme is expected to achieve high detection reliability and to minimize energy consumption by turning off the transmit-receive antenna pair which is affected from deep fading.

\section{B. Adaptive Signal Transmission Scheme}

In order to enable adequate decisions on the selection of appropriate optimization scheme adaptively, link adaptation mechanisms require knowledge of the required transmission quality over the given channel conditions. Generally, the transmission quality is based on frame error probability conditioned on the particular realization of the channel, but such information is not accessible directly. Hence, it arises the need to define a measure that maps directly to the frame error probability which is defined as channel quality index (CQI). CQI is designed in a manner to ensure robustness against signal distortions caused by the propagation and interference conditions of the channel as well as to guarantee the optimized utilization of resources while maintaining required quality of service. In this paper, a measure of CQI is proposed so that the link between transmitter and receiver is maintained for a given quality of service. The proposed measure of CQI is defined as:

$$
C Q I=f\left(E\left[(\vec{\lambda}-\mu)^{2}\right]\right)
$$

Where

$$
\mu=E[\vec{\lambda}]
$$

Where $E[\cdot]$ represents expectation value and $\vec{\lambda}$ is a set of eigen vctor of dimension $\left(N_{r} \times 1\right)$ which is defined as:

$$
\vec{\lambda}=\left[\lambda_{1}, \lambda_{2}, \ldots, \lambda_{N_{r}}\right]^{T}
$$

Where $\lambda(\cdot)$ represents the eigen values of channel coefficients. The selection of transmission 
scheme is proposed to be based on the classification of propagation condition, which can be obtain from the list of CQI as presented in Table II. CQI is described based on the condition of the channel indexed from 0 to 3 . The higher index represents the higher requirement of cooperation to maintain the required link reliability.

TABLE II

\begin{tabular}{|c|c|c|c|c|}
\hline CQI & $\mathbf{0}$ & 1 & 2 & 3 \\
\hline $\begin{array}{c}\text { Normalized Channel } \\
\text { Quality Measure }\end{array}$ & $<0.4$ & $0.4-0.6$ & $0.6-0.75$ & $>0.75$ \\
\hline $\begin{array}{c}\text { Selection of } \\
\text { Transmission Scheme }\end{array}$ & $\begin{array}{l}\text { Conventional } \\
\text { Cooperation } \\
\text { Scheme }\end{array}$ & $\begin{array}{c}\text { Proposed } \\
\text { Resource Selection } \\
\text { Scheme }\end{array}$ & $\begin{array}{l}\text { Proposed } \\
\text { Hybrid } \\
\text { Scheme }\end{array}$ & $\begin{array}{c}\text { Lattice } \\
\text { Reduction }\end{array}$ \\
\hline
\end{tabular}

\section{SIMULATION AND RESULTS}

To evaluate the performance of the proposed cooperative transmission scheme, a collaborative wireless sensor network with Rayleigh fading channel is considered. The number of sensing nodes within a cluster is bounded with a range set of $n_{p} \in\{3: 5\}$ in our simulation. It is assumed that multiple sensor nodes cooperate while transmitting sensed data. The fusion center is assumed to be equipped with multiple antennas to act as a virtual multiple-input multiple-output system, while receiving signal from multiple sensing nodes. It is assumed that the channel remains constant during each transmission frame. Binary phase shift keying (BPSK) and quadrature phase shift keying (QPSK) modulation schemes are considered for simplicity.

A set of sensing nodes within three neighbouring clusters has been considered. The fusion center is assumed to receive data from the sensing nodes with three antennas. Hence $N_{t}=N_{r}=3$ appears in our simulation model. For the ease of implementation Zero Forcing (ZF) and Minimum Mean Square Error (MMSE) detectors have been considered at the fusion center receiver. It is expected that adaptive resource selection scheme will reduce energy consumption by turning off the transmit-receive antenna pair which is affected by 
deep fading. Fig. 3 presents with a comparative performance study of the proposed adaptive resource selection and hybrid transmission schemes over conventional cooperative schemes (conventional virtual MIMO) and existing adaptive transmission scheme (lattice reduction) as found in the literature. It is observed from Fig. 3 that adaptive resource selection scheme increases detection reliability as compared to conventional cooperative MIMO scheme. It is also observed that lattice reduction scheme achieves the highest detection reliability among the rest of the other schemes. However lattice reduction requires significant computational intensity within the available resources, hence its not energy efficient. The proposed hybrid scheme is expected to provide a tradeoff between energy efficiency and detection reliability as evident in Fig. 3.

As mentioned in section III, an accurate measure of CQI is the key to the performance of the proposed adaptive resource allocation in energy efficient collaborative transmission. In order to select appropriate optimization scheme adaptively based on the information from fusion center through a feedback link, a measure of CQI has been proposed in Eq. 9 and its normalized behaviour has been realized in Fig. 4. Table II presents resource allocation decision boundaries of CQI values which has been considered to select appropriate transmission scheme. It is expected that the proposed adaptive transmission scheme achieves higher energy efficiency and link reliability while maintaining required quality of service. Fig. 5 presents the performance comparison between the proposed adaptive transmission scheme and hybrid scheme for $N_{t}=$ $N_{r}=3$ while CQI based transmission scheme selection has been implemented. It is observed that the proposed adaptive transmission scheme and the proposed hybrid scheme outperformed the conventional cooperative transmission scheme by $8.5 d B$ and $7.5 d B$ respectively for a given Bit Error Rate (BER) of $10^{-3}$, where $N_{t}=N_{r}=3$. Fig. 6 and Fig. 7 present the performance comparison of the proposed CQI based adaptive transmission, hybrid and adaptive resource selection schemes with conventional cooperative transmission and lattice reduction schemes. QPSK modulation scheme is considered with transmission dimension $N_{t}=N_{r}=3$ 
to generate the results in Fig. 6 and Fig. 7, while ZF and MMSE detection has been exploited respectively. It is observed from Fig. 8 that the proposed adaptive transmission, hybrid and adaptive resource selection schemes outperforms the conventional cooperative transmission scheme for $N_{t}=N_{r}=4$.

\section{CONCLUSiON}

Considering the energy constrains within green theme based communication, an adaptation criterion based resource selection model is proposed. Within collaborative wireless sensor network, a set of cooperative transmission frameworks have been proposed. The basis of adaptation criterion is a perfect estimate of the channel state information at the receiver, which has been assumed to be fed back to the transmitter. The proposed hybrid scheme achieves the required detection reliability with significantly lower energy requirement compared to its existing counterparts. Besides this, with the expense of a set of negligible computational complexity, the proposed adaptive transmission scheme is found to be able to save additional energy requirement while providing the same detection reliability. For the future extension of this work, authors are intended to extend this work for asymmetric transmit-receive antennas.

\section{REFERENCES}

[1] Y. Wei, M. Song, B. Liu, X. Wang, and Y. Li, "Energy efficient cooperative relaying and cognitive radio technologies to deliver green communication," in Pervasive Computing and Applications (ICPCA), 2011 6th International Conference on, 2011, pp. 105-109.

[2] A. Amokrane, R. Langar, R. Boutaba, and G. Pujolle, "A green framework for energy efficient management in tdmabased wireless mesh networks," in Network and service management (cnsm), 2012 8th international conference and 2012 workshop on systems virtualiztion management (svm), 2012, pp. 322-328.

[3] G. Anastasi, M. Conti, M. Di Francesco, and A. Passarella, "Energy conservation in wireless sensor networks: A survey," Ad Hoc Networks, vol. 7, no. 3, pp. 537-568, 2009.

[4] I. Akyildiz, W. Su, Y. Sankarasubramaniam, and E. Cayirci, "A survey on sensor networks," IEEE Communications Magazine, vol. 40, no. 8, pp. 102 - 114, Aug 2002.

[5] F. Zhao, J. Shin, and J. Reich, "Information-driven dynamic sensor collaboration for tracking applications," IEEE Signal processing magazine, vol. 19, no. 2, pp. 61-72, 2002.

[6] C.-Y. Shih and S. Jenks, "A dynamic cluster formation algorithm for collaborative information processing in wireless sensor networks," in 3rd International Conference on Intelligent Sensors, Sensor Networks and Information, dec. 2007, pp. $107-112$.

[7] J. Fang and H. Li, "Power constrained distributed estimation with cluster-based sensor collaboration," IEEE Transactions on Wireless Communications, vol. 8, no. 7, pp. 3822 -3832, july 2009.

[8] S. Hashmi, H. Mouftah, and N. Georganas, "Achieving reliability over cluster-based wireless sensor networks using backup cluster heads," in IEEE Global Telecommunications Conference, 2007. GLOBECOM, nov. 2007, pp. 1149 1153.

[9] V. Tarokh, N. Seshadri, and A. Calderbank, "Space-time codes for high data rate wireless communication: performance criterion and code construction," IEEE Transactions on Information Theory, vol. 44, no. 2, pp. 744 -765, Mar 1998. 
[10] G. Miao, Signal Processing for Digital Communications: Theory, Algorithms And Applications. Artech House, Inc. Norwood, MA, USA, 2006.

[11] G. Venkataraman, S. Emmanuel, and S. Thambipillai, "Energy-efficient cluster-based scheme for failure management in sensor networks," IET Communications, vol. 2, no. 4, pp. 528-537, 2008.

[12] A. Molisch, M. Steinbauer, M. Toeltsch, E. Bonek, and R. Thoma, "Capacity of MIMO systems based on measured wireless channels," IEEE Journal on Selected Areas in Communications, vol. 20, no. 3, pp. 561 -569, Apr 2002.

[13] V. Tarokh, N. Seshadri, and A. Calderbank, "Space-time codes for high data rate wireless communication: performance criterion and code construction," IEEE Transactions on Information Theory, vol. 44, no. 2, pp. 744 -765, Mar 1998.

[14] L. Zheng and D. Tse, "Diversity and multiplexing: a fundamental tradeoff in multiple-antenna channels," IEEE Transactions on Information Theory, vol. 49, no. 5, pp. 1073 - 1096, May 2003.

[15] E. Telatar, "Capacity of multi-antenna Gaussian channels," European transactions on telecommunications, vol. 10, no. 6, pp. 585-595, 1999.

[16] G. Barriac, R. Mudumbai, and U. Madhow, "Distributed beamforming for information transfer in sensor networks," in Third International Symposium on Information Processing in Sensor Networks, april 2004, pp. $81-88$.

[17] J. Adeane, M. R. D. Rodrigues, and I. Wassell, "Lattice-reduction-aided detection for mimo-ofdm-cdm communication systems," Communications, IET, vol. 1, no. 3, pp. 526-531, 2007.

[18] X. Wu and J. Thompson, "Accelerated sphere decoding for multipleinput multiple-output systems using an adaptive statistical threshold," Signal Processing, IET, vol. 3, no. 6, pp. 433-444, 2009.

[19] Y. Zhang, "Effective reduction for sphere decoder in linear multi-input multi-output channel systems," Communications, IET, vol. 6, no. 11, pp. 1573-1578, 2012.

[20] X. Ma and W. Zhang, "Performance analysis for mimo systems with lattice-reduction aided linear equalization," IEEE Transactions on Communications, vol. 56, no. 2, pp. 309 -318, february 2008.

[21] J.-M. Chung, J. Kim, and D. Han, "Multihop hybrid virtual mimo scheme for wireless sensor networks," Vehicular Technology, IEEE Transactions on, vol. 61, no. 9, pp. 4069-4078, 2012.

[22] S. Jayaweera, "Energy efficient virtual mimo-based cooperative communications for wireless sensor networks," in Proceedings of 2005 International Conference on Intelligent Sensing and Information Processing, 2005., jan. 2005, pp. $1-6$.

[23] R. Mudumbai, G. Barriac, and U. Madhow, "On the feasibility of distributed beamforming in wireless networks," IEEE Transactions on Wireless Communications, vol. 6, no. 5, pp. 1754 -1763, may 2007.

[24] R. Mudumbai, D. Brown, U. Madhow, and H. Poor, "Distributed transmit beamforming: challenges and recent progress," IEEE Communications Magazine, vol. 47, no. 2, pp. 102 -110, february 2009.

[25] C.-W. Chang, A. Kothari, A. Jafri, D. Koutsonikolas, D. Peroulis, and Y. Hu, "Radiating sensor selection for distributed beamforming in wireless sensor networks," in Military Communications Conference, 2008. MILCOM 2008. IEEE, 2008, pp. 1-7.

[26] Z. Han and H. Poor, "Lifetime improvement in wireless sensor networks via collaborative beamforming and cooperative transmission," Microwaves, Antennas Propagation, IET, vol. 1, no. 6, pp. $1103-1110$, dec. 2007.

[27] M. Elfituri, A. Ghrayeb, and W. Hamouda, "Antenna/relay selection for coded wireless cooperative networks," in IEEE International Conference on Communications, may 2008, pp. 840 -844.

[28] Y. Zhang, Y. Cai, W. Yang, and Y. Xu, "An energy-efficient cooperative node selction scheme in wireless sensor networks," in International Conference on Communication Software and Networks, ICCSN '09., feb. 2009, pp. 232 $-236$. 


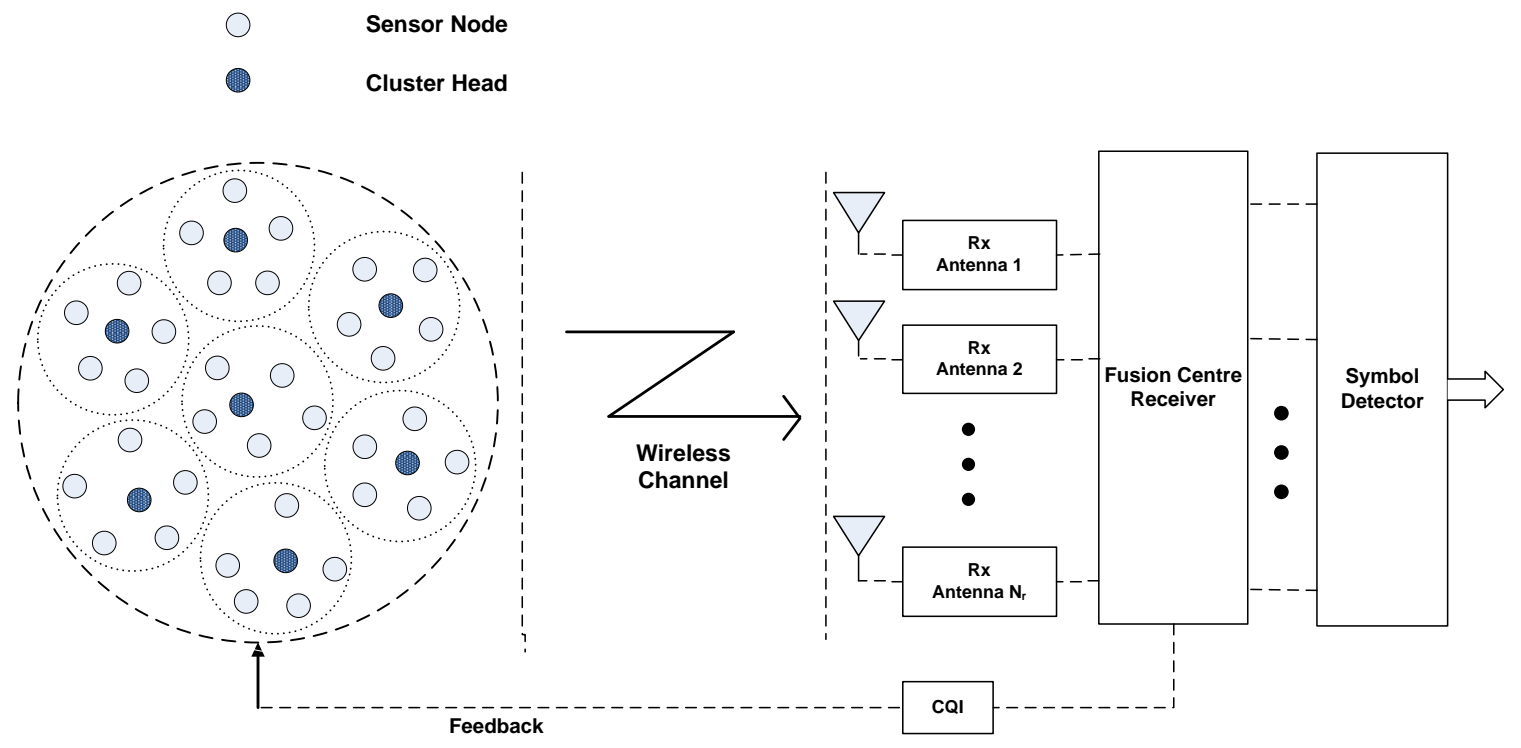

Fig. 1. Typical communication model within wireless sensor network 


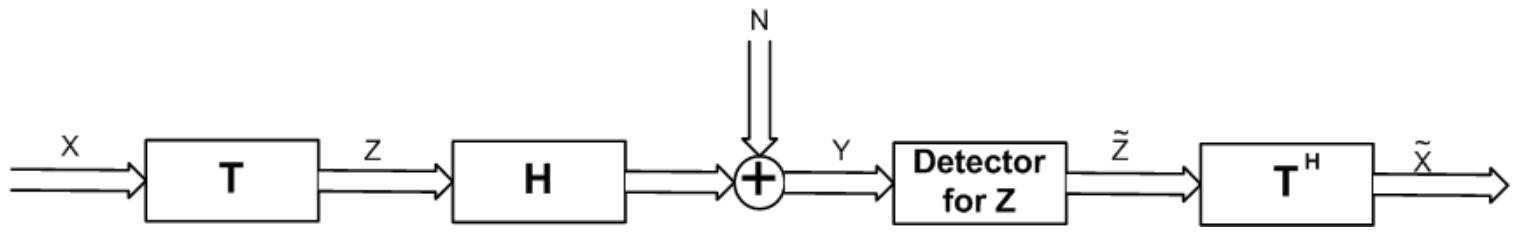

Fig. 2. Block Diagram for a MIMO system with Lattice Reduction Aided data detection 


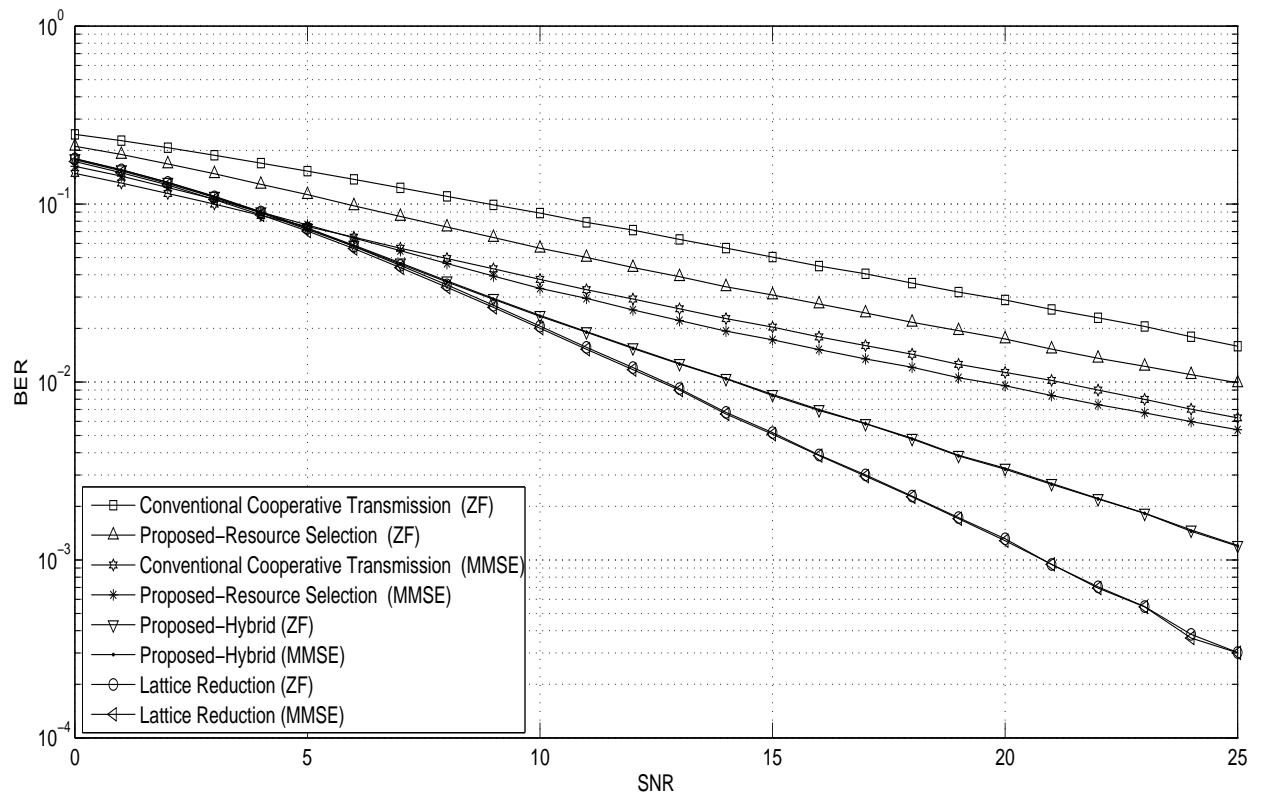

Fig. 3. Performance comparison of the proposed hybrid scheme and antenna selection scheme with conventional cooperative transmission scheme and Lattice Reduction (LR) for Zero Forcing (ZF) and Minimum Mean Square Error (MMSE) detection schemes where number of transmit and receive antennas are 3 and modulation scheme is Binary Phase Shift Keying (BPSK) 


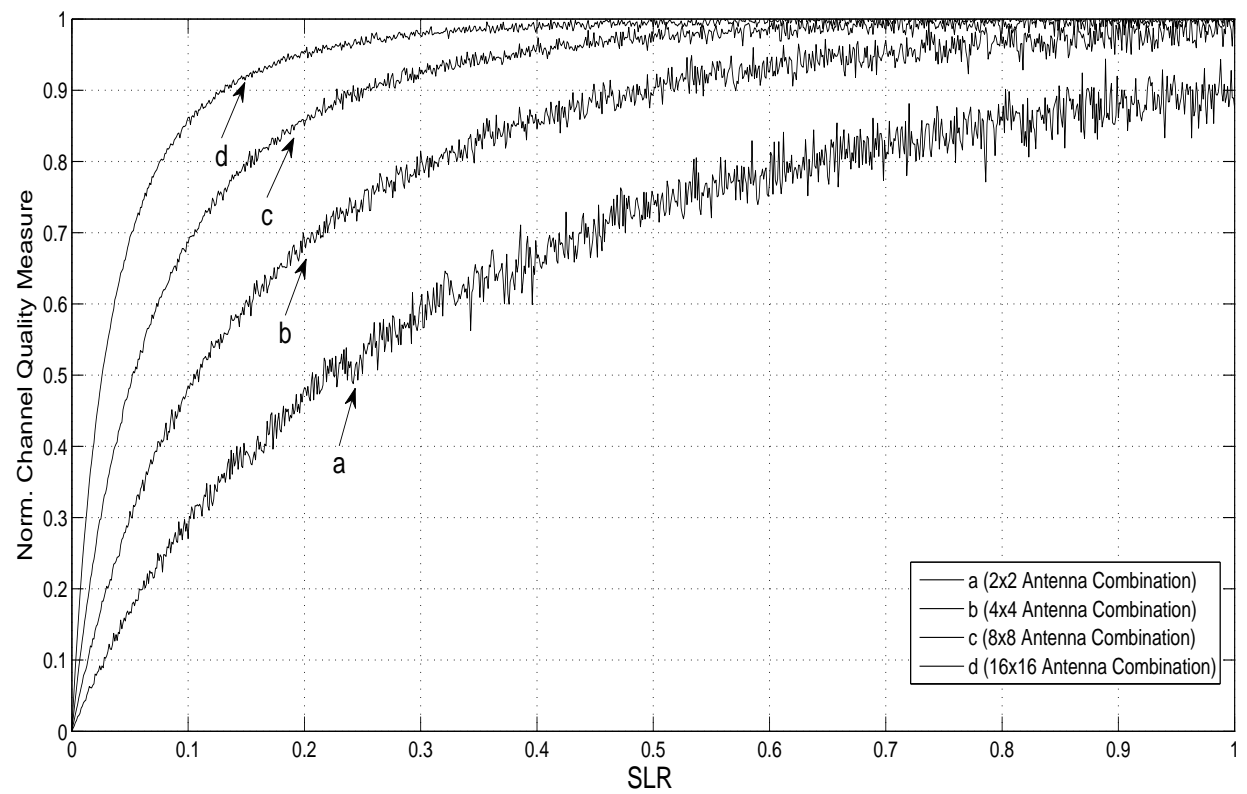

Fig. 4. Proposed normalized channel quality measure for transmit-receive antenna combinations of 2, 4, 6 and 8 


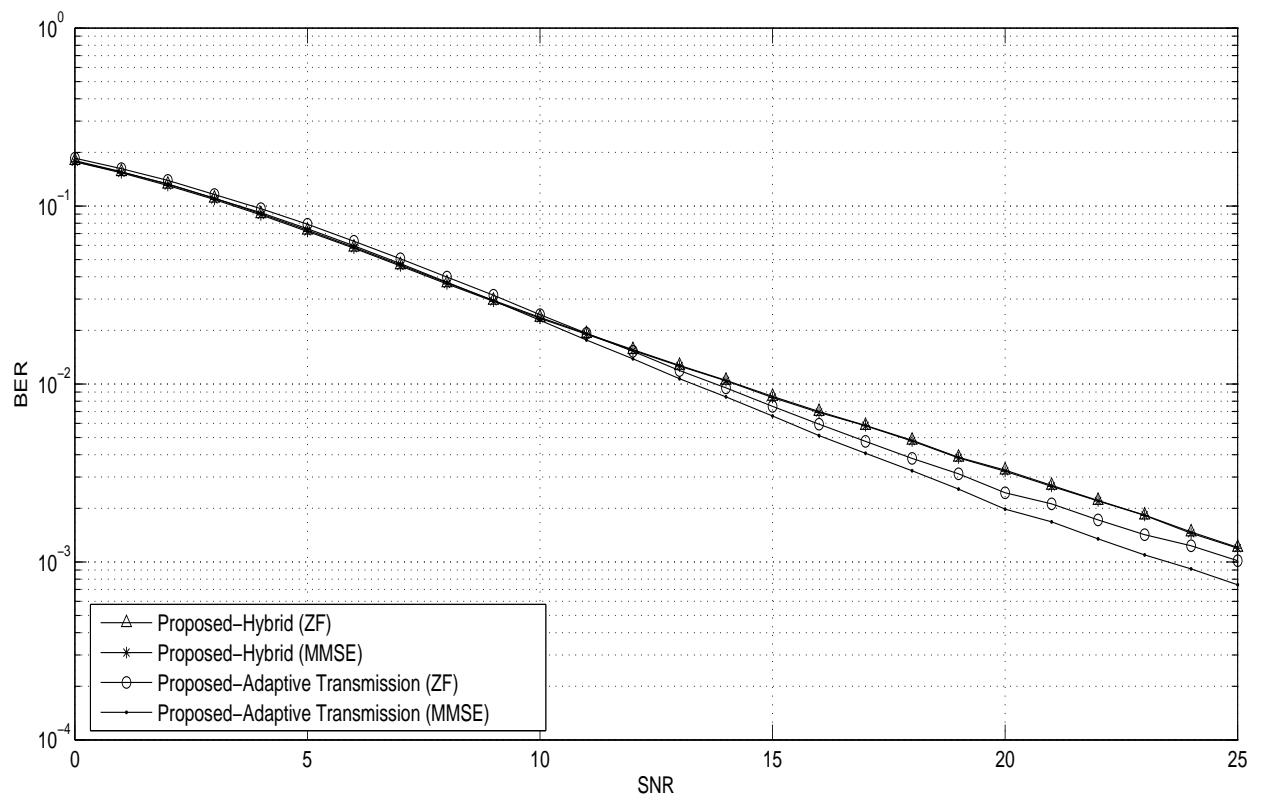

Fig. 5. Performance comparison of the proposed hybrid scheme and proposed adaptive transmission scheme for Zero Forcing (ZF) and Minimum Mean Square Error (MMSE) detection schemes with transmit and receive antennas are 3 and modulation scheme is Binary Phase Shift Keying (BPSK) 


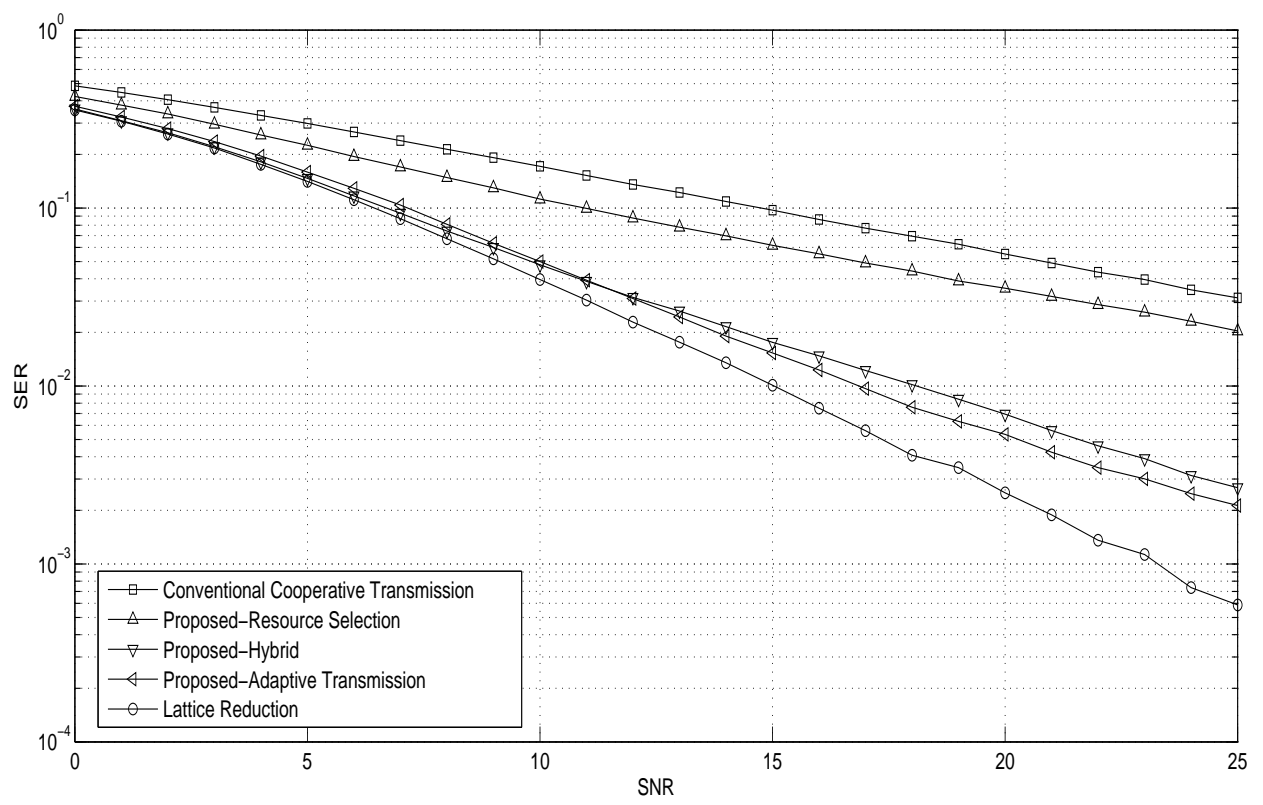

Fig. 6. Performance comparison of the proposed adaptive transmission, antenna selection and hybrid schemes with Lattice Reduction (LR) and conventional cooperative transmission schemes for Zero Forcing (ZF) detection scheme with transmit and receive antennas are 3 and modulation scheme is Quadrature Phase Shift Keying (QPSK) 


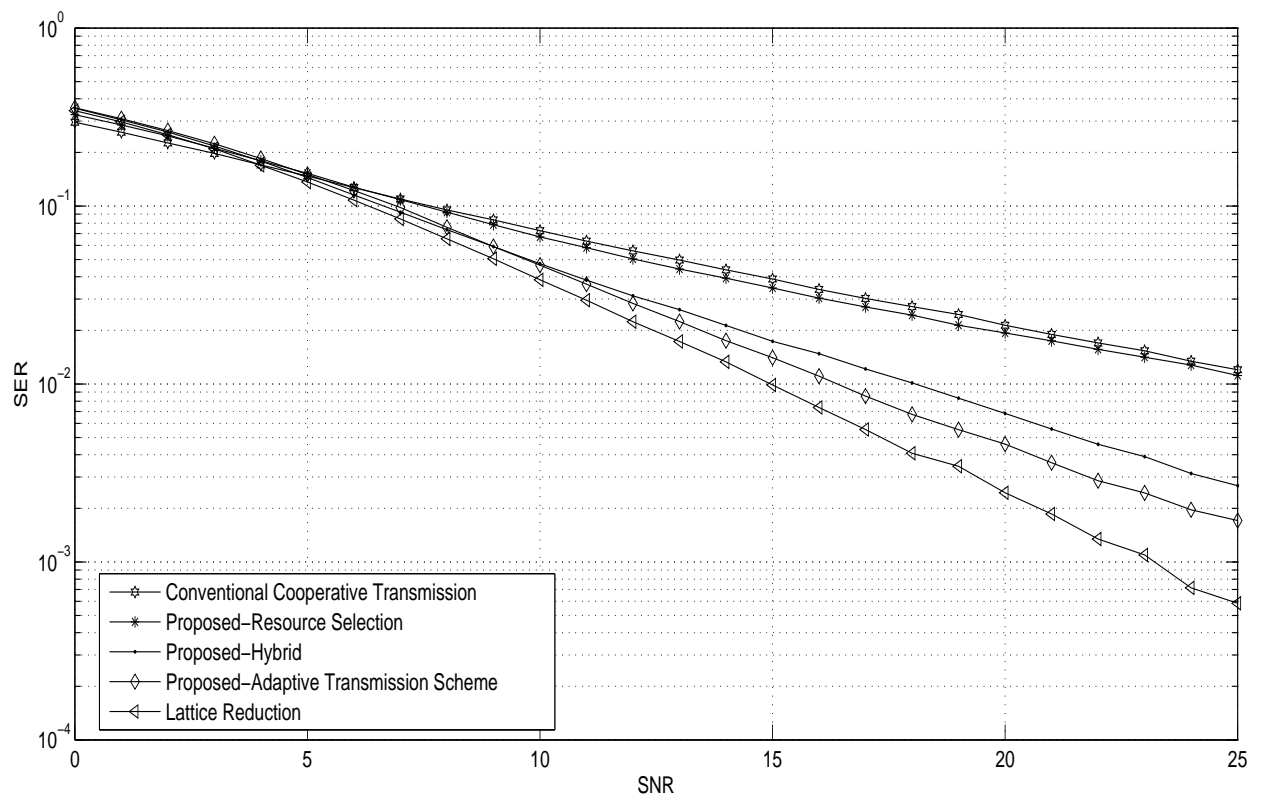

Fig. 7. Performance comparison of the proposed adaptive transmission, antenna selection and hybrid schemes with Lattice Reduction (LR) and conventional cooperative transmission schemes for Minimum Mean Square Error (MMSE) detection scheme with transmit and receive antennas are 3 and modulation scheme is Quadrature Phase Shift Keying (QPSK) 


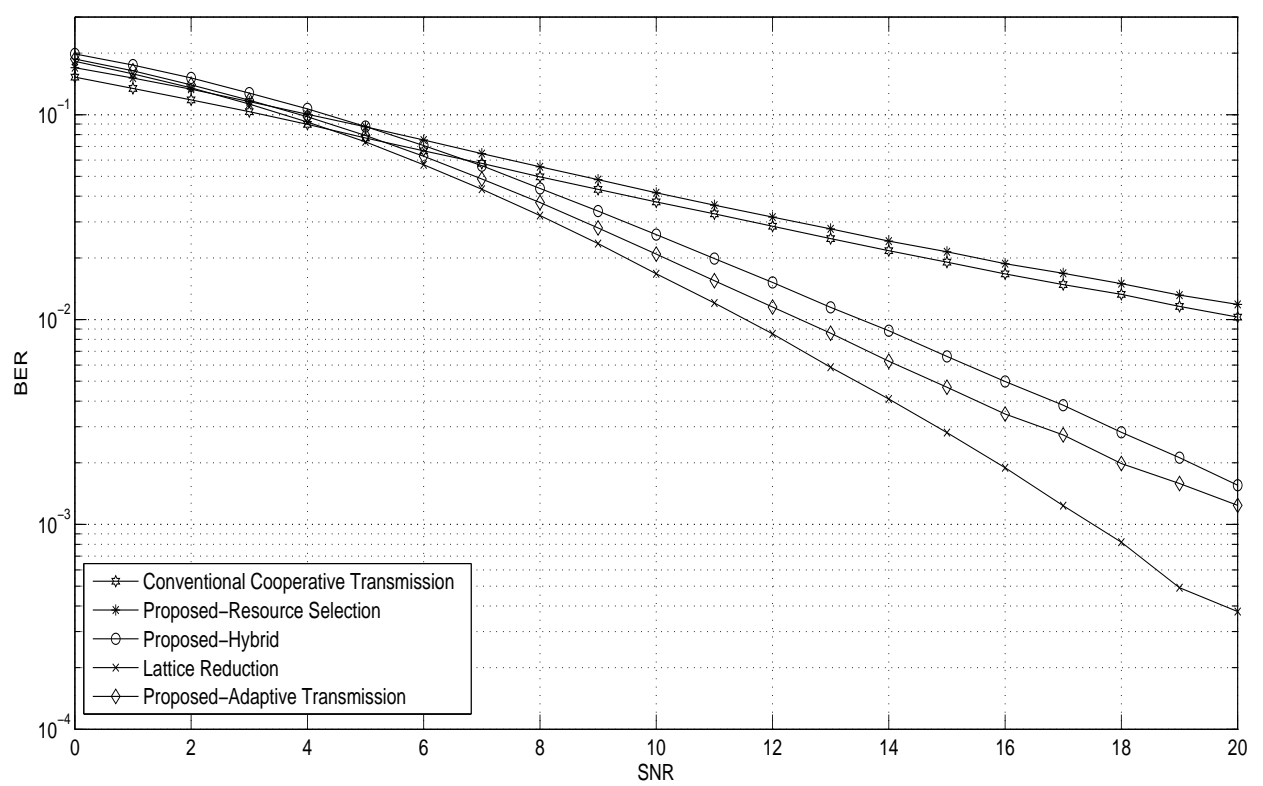

Fig. 8. Performance comparison of the proposed adaptive transmission, antenna selection and hybrid schemes with Lattice Reduction (LR) and conventional cooperative transmission schemes for Minimum Mean Square Error (MMSE) detection scheme with transmit and receive antennas are 4 and modulation scheme is Binary Phase Shift Keying (BPSK) 